\title{
Research on Evaluation of CAM Image Segmentation Algorithms on a new Database
}

\author{
Yongfeng Huang ${ }^{1, \mathrm{a}}$, Zhihan Zhang ${ }^{1, \mathrm{~b}}$,Cairong Yan ${ }^{1, \mathrm{c}}$ and Qi Lu ${ }^{1, \mathrm{~d}}$ \\ ${ }^{1}$ School of Computer Science and Technology, Donghua University, \\ Shanghai 201620, China

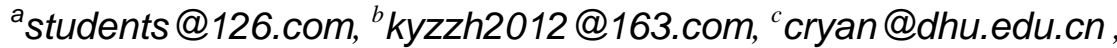 \\ dluqi_alice@foxmail.com
}

\begin{abstract}
The segmentation of CAM images are prerequisite and fundamental steps of studying the drug effects on angiogenesis. In this paper, an evaluation method for different segmentation algorithm of CAM images is proposed. Firstly, a CAM images dataset consists of 4 distinctive features (uneven illumination, vascular rupture, vascular disconnection and tiny vessels) is set up in order to study the performance of various CAM segmentation algorithms. Then, detailed comparisons are made among 4 classical segmentation algorithms, by comparing their outputs to our standard manual segmentation results of the dataset. Results show that the proposed evaluation method can accurately and sensitively determine the performance of these classical algorithms for CAM blood vessels under various conditions.
\end{abstract}

Keywords: CAM, segmentation evaluation, image segmentation, image set

\section{Introduction}

Cancer has a close relationship with angiogenesis [1]. By tracking the activities of angiogenesis the tumor growth and inhibition can be evaluated. The chicken chorioallantoic membrane is a common experiment carrier of angiogenesis activity. With CAM [2-4] image segmentation the measurement on surface area, length and number of branches of the vessel of the determined area can be achieved, which therefore can evaluate changes in blood vessel growth activities. However, the CAM image segmentation result is the basis of assessing accurately angiogenesis.

Although there are a lot of CAM-related blood vessel extraction algorithms, not every specific algorithm for every specific images have such a high degree of recognition. However, a problem arises. How to choose the appropriate image segmentation algorithm to recognize specific images? A unified system should be set up to evaluate the accuracy of different algorithms for image recognition. In the evaluation method of image segmentation, according to the research field of image, different image libraries are usually designed to review the pros and cons of different segmentation algorithms, such as: the retina's STARE database 
[5-6], DRIVE database [7], FVC2002 and FVC2004 database [8] of fingerprint identification, CMU- PIE database [9], FERET database [10] for evaluating the face recognition, and so on.

On the basis of this, this paper mainly studies the four factors influencing the CAM image segmentation, set up four image set of CAM test image library. By using the method of difference experiment evaluation of medical image segmentation, the author proposed a method to evaluate CAM blood vessels segmentation algorithm. This method can evaluate the accuracy and sensitivity of algorithm to recognize the four factors of CAM image---uneven illumination, partial rupture blood vessel, local disconnection blood vessels and tiny blood vessels.

\section{CAM image database and evaluation method}

CAM is a qualitative and quantitative research techniques in vivo angiogenesis. In most cases, with the help of a high numerical aperture and fast electroniccoupled device (CCD) CAM can sample the direct CAM image, split the vascular area from the CAM image and make a data analysis for segmented image and then evaluate the situation of angiogenesis with the statistical results of the number of branch, area and length of blood vessels. However, that CAM segmentation results is good or not directly affect the quality of the data analysis that follows.

There are many factors that can influence the effect of CAM image segmentation, which can be mainly summarized in four categories: uneven illumination when sampling, local vascular disconnection caused by manual operation when preparing sample, local vascular rupture and difficult definition of tiny blood vessels caused by lack of precision of sampling, which are shown in Figure 1.In this paper, based on four main factors that influence CAM image segmentation the author organized these experimental images into four image sets. They are respectively uneven illumination image set, local vascular disconnection image set, local vascular rupture image set and tiny blood vessels image set. These four CAM image sets form a database for the CAM image segmentation algorithm test. Each image set is composed of 12 pairs of slices and corresponding reference diagram, of which the reference diagram is based on the figure carved out by the biology experts and can be usually regarded as "Gold standard" of the split results.

\subsection{CAM image database}

Uneven illumination image set. There is a lot of uneven illumination of CAM image. This uneven illumination is shown as: low gray value of the image as a whole, local vascular gray value is too low and the local background brightness of the entire image is too strong. Gray value of the image as a whole is too low mainly due to the ambient poor light conditions or the poor device itself in the process of image acquisition. Therefore, the overall gray value of images obtained and the image contrast are both quite low. It is also difficult to identify 
a region of interest; Local vascular gray value is too low mainly caused by insufficient light acquisition or lower grey values located in the gray shaded area and integrated with backgrounds together; the brightness of local image background is too strong. It leads to the decrease of blood vessels gray value, which is close to that of background and it is difficult to identify. In a word, the above images with uneven illumination characteristics can be organized into a set of images, which is shown in figure 1 (a). This set of images can be used to assess robustness of algorithm on uneven illumination conditions.

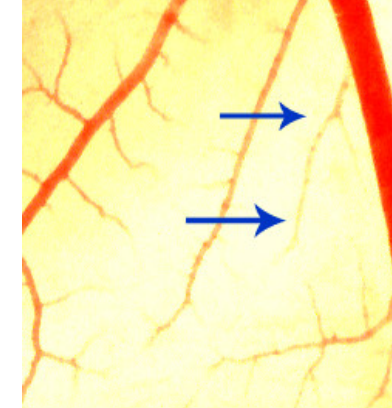

(a)

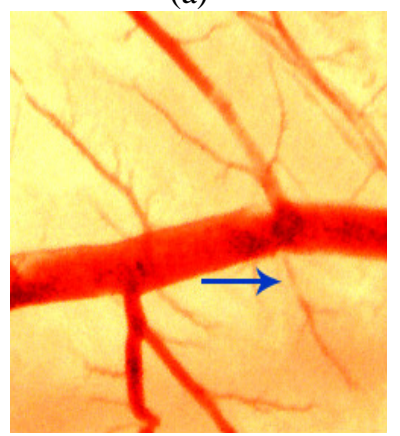

(c)

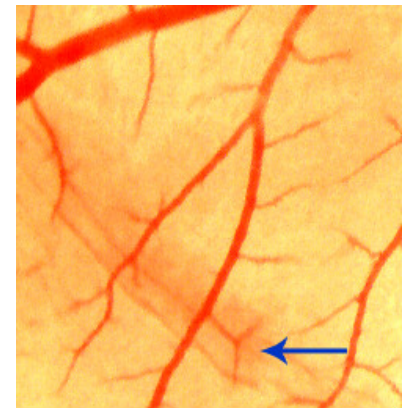

(b)

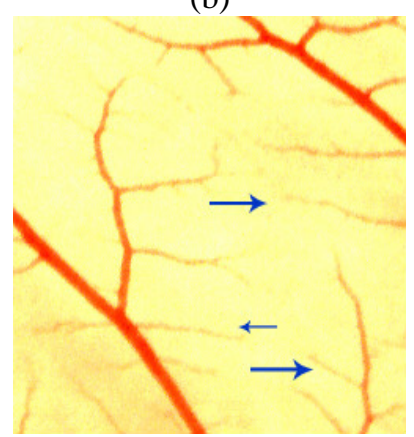

(d)

Fig.1 Four CAM images of different feature.

(a) Uneven illumination image, (b) Local vascular rupture image

(c)Local vascular disconnection image, (d) Tiny vascular image

Local vascular rupture image set. In the process of CAM image acquisition, it is inevitable to perform physical operation to remove the shell of the CAM, which can lead to a partial breakdown of blood vessels, to the diffusion of the plasma to nearby areas. Blood vessels and their background seem a blur. It may be easy for researchers to mistake a large number of local blood clot area as a target vessel. Figure 1 (b) shows the partial breakdown of blood vessels infiltrate into the surrounding vascular tissue. Local vascular breakdown image set can be used to assess robustness of algorithm on non-vascular area detection.

Local vascular disconnection image set. In CAM blood vessels image, due to other tissues covering of blood vessels, the camera angles and other causes, blood vessels in photos exist disconnection, which results in an incomplete blood vessel. Therefore, the extracted blood vessels lost their integrity and the accuracy of the following data analysis will accordingly decrease. From figure 1(c), we 
can find out the main blood vessel and the surrounding blood vessels are all broken and disconnection. It is easy for us to make identification that they come form different vessels. Therefore, whether CAM image can detect the disconnection blood vessels and at the same time keep its integrity is an important indicator to evaluate segmentation algorithm.

Tiny blood vessels image set. In CAM images there are both main thick blood vessels and a large number of small blood vessels. These main blood vessels are easier to be identified due to their bulky diameter and high contrast. On the contrary, small blood vessel's diameter is smaller, and the contrast with the background is lower. Therefore, it is difficult to identify. Meanwhile, the small blood vessels and main vascular have a large gray span and contain many nonvascular impurities, which also increase the difficulty of its identification. Small blood vessels show the richness of blood vessels. If there is lack of the tiny blood vessels, the recognition accuracy and integrity will be discounted. As a result, the accuracy of extracting tiny blood vessels is also an important part of evaluating segmentation algorithm. Figure 1 (d) is one picture of tiny blood vessels image set.

\subsection{Evaluation methodology}

Research on evaluation method of image segmentation, on one hand, can improve the performance of the existing algorithm, and on the other hand, can be of great significance to guide new segmentation technology research. By far, there has not been a recognized objective standard to judge whether digital image segmentation results are satisfactory or not. For specific CAM image segmentation, this paper, based on CAM image library previously proposed and combined with the difference experiment method of medical segmentation evaluation index, puts forward a set of comprehensive evaluation method for CAM vessel segmentation algorithm.

The author uses pixel-based statistics to measure and evaluate each single image. Each pixel is marked as object or background. By comparing with pixels in standard reference image, the pixels in the image for evaluation can be divided into the following four types: true positive (TP), false positives (FP), true negative (TN) and false negatives (FN).

Suppose that $\mathrm{I}$ is the entire image, $\mathrm{G}$ is the set of target pixel in the standard reference image, and $\mathrm{R}$ is the set of the target pixels for evaluation obtained by the segmentation algorithm. The above four types is expressed as:

$$
\begin{aligned}
T P & =R \cap G \\
\mathrm{FP} & =\mathrm{R}-\mathrm{G} \\
\mathrm{TN} & =\mathrm{I}-\mathrm{G}-\mathrm{R} \\
\mathrm{FN} & =\mathrm{G}-\mathrm{R}
\end{aligned}
$$

Pixel-based statistical evaluation is described by three indicators, namely AR, FNR and FPR [11]. Accuracy (AR) represents the ratio of all correctly classified pixels, that is: 


$$
\mathrm{AR}=\frac{T P+T N}{T P+F P+T N+F N}
$$

FNR is expressed as:

$$
F N R=\frac{F N}{G}
$$

FPR is expressed as:

$$
F P R=\frac{F P}{G}
$$

For single image, the above-mentioned three indicators can obtain from each image segmentation result. However, for the indicator of each image set we need to get the statistics of each image indicator in each image set. The comprehensive evaluation indicator of overall image set is defined as:

$$
R_{k}=\frac{1}{M} \frac{1}{N} \sum_{i=1}^{N} \sum_{j=1}^{M} X_{k i j}
$$

In the above equation, $R_{k}$ represents a comprehensive evaluation of the $K$ th algorithm. Here it refers to the above three indicators. i represents the number of image sets, and here the number of the above-mentioned image set is four, $N=4$. j refers to the number of images in the image set, $M=12$. $X_{k i j}$ represents the $k$-th indicator of the $j$-th image in $i$-th image set. The indicator of each image set is the average of all image indicators. And comprehensive indicator is the average of all image set indicators.

\section{Experiments and results}

\subsection{Experiments}

The CAM segmentation algorithm is mainly based on the supervised learning and threshold. Hufen, Xuzhongyu [12] propose a new image processing method based on support vector machine by image texture feature extraction. Charalampos N. Doukas [13] propose an adaptive local threshold segmentation algorithm. Hoover [5] proposed a matched filter based on different direction and scale to segment the vascular. While Mendonca [14] introduces a region growing method to form the final segmentation result after processed by a specific designed filter

In this paper, the above four kinds of segmentation methods have been tested by using the proposed evaluation methods based the database of CAM image.

\subsection{Results}

Table 1 shows an overview of the parameter index (AR, FNR, FPR) of the segmentation methods on four image sets.

Figure 2 shows the AR of the four segmentation methods on four different influence factors. The AR of Mendonca and SVM method are significantly higher than that of Hoover and Charalampos method, the AR of Hoover is slightly higher than Charalampos method. In addition, the AR of Mendonca and 
SVM method have been greatly improved on the tiny image set comparing with the Hoover and Charalampos method. This shows that the recognition of Mendonca and SVM method are superior compared with the other two methods under the tiny vascular influence factor. As to the Mendonca and SVM method, it has a similar recognition accuracy among the four influence factor. While the AR of Hoover on the tiny vascular and local rupture set is far less than the other two vascular set, it indicates that Hoover method is inefficient to the detection of small blood vessels and local rapture. The Charalampos method have a poor accuracy on the four image sets, especially on the tiny blood vessels set, uneven illumination set and ruptured blood vessels set.

Table 1 Experiment result of four kinds of algorithms

\begin{tabular}{cccccc}
\hline & Methods & Tiny & Illumination & Disconnection & Rupture \\
\hline \multirow{4}{*}{ AR } & Charalampos & 0.7887 & 0.8127 & 0.8205 & 0.8225 \\
& SVM & 0.8513 & 0.8294 & 0.8330 & 0.8357 \\
& Hoover & 0.7973 & 0.8213 & 0.8291 & 0.8311 \\
& Mendonca & 0.8488 & 0.8269 & 0.8305 & 0.8332 \\
\hline \multirow{3}{*}{ FN } & Charalampos & 0.2179 & 0.2222 & 0.2197 & 0.2659 \\
R & SVM & 0.1881 & 0.1991 & 0.1962 & 0.2154 \\
& Hoover & 0.2152 & 0.2254 & 0.2135 & 0.2316 \\
& Mendonca & 0.1961 & 0.2073 & 0.1976 & 0.2178 \\
\multirow{4}{*}{ FPR } & Charalampos & 0.1839 & 0.1533 & 0.1398 & 0.1363 \\
& SVM & 0.1346 & 0.1292 & 0.1189 & 0.1174 \\
& Hoover & 0.1741 & 0.1426 & 0.1285 & 0.1279 \\
\hline
\end{tabular}

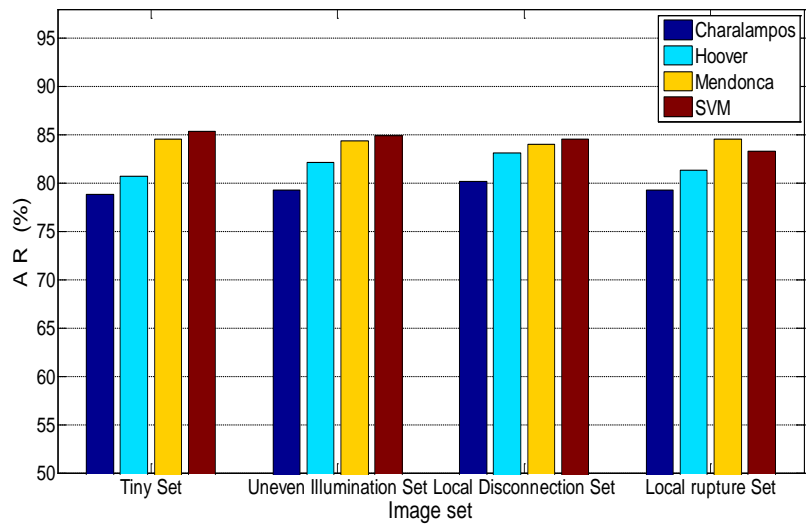

Fig.2 Comparison of the AR on four image sets

Figure 3 shows the FNR of the four segmentation methods on four different influence factors. The higher of the FNR value is, the worse the performance of the algorithm. The FPR of Hoover and Charalampos method are significantly higher than that of Mendonca and SVM method. A considerable number of 
vascular have not been distinguished that mainly concentrated on the missing of large small blood vessels or the influence by the uneven illumination. Although the Hoover method is superior compared with the Charalampos method, it still can't largely improve the recognition rate of small blood vessels. The four different algorithms have a similar effect on the local vascular disconnection set and Local vascular rupture image sets.

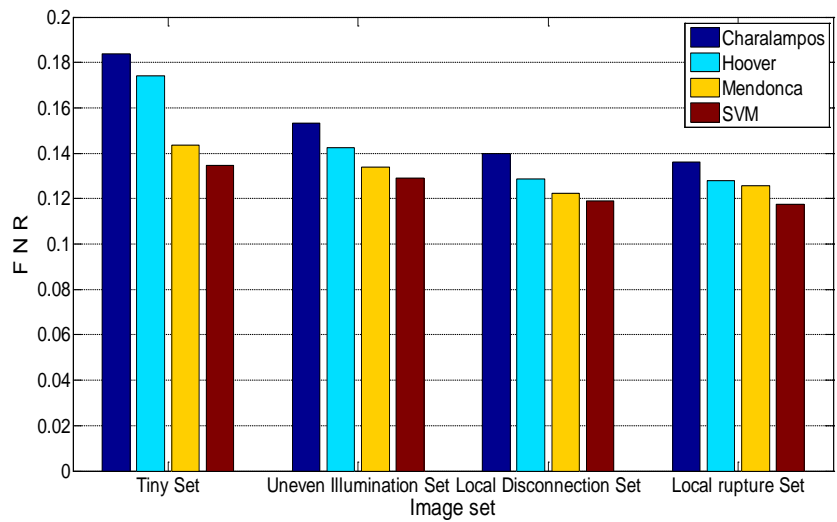

Fig.3 Comparison of the FNR on four image sets

Figure 4 shows the FPR value of the four segmentation methods on four different influence factors. Although the recognition accuracy of Hoover, Mendonca and SVM is better than the Charalampos method, but the four methods is not excellent to achieve the desirable effect. The four algorithms have a high FPR on local rapture set.

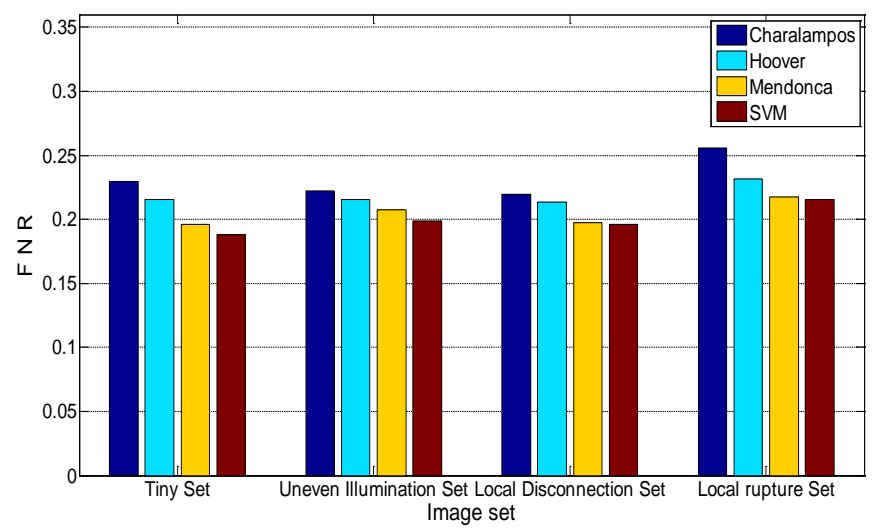

Fig.4 Comparison of the FPR on four image sets

Through the analysis of the above experimental results, the sensitivity of the algorithms to different factors can be tracked obviously. Figure 5 shows the influence of the four segmentation methods on four different influence factors. 


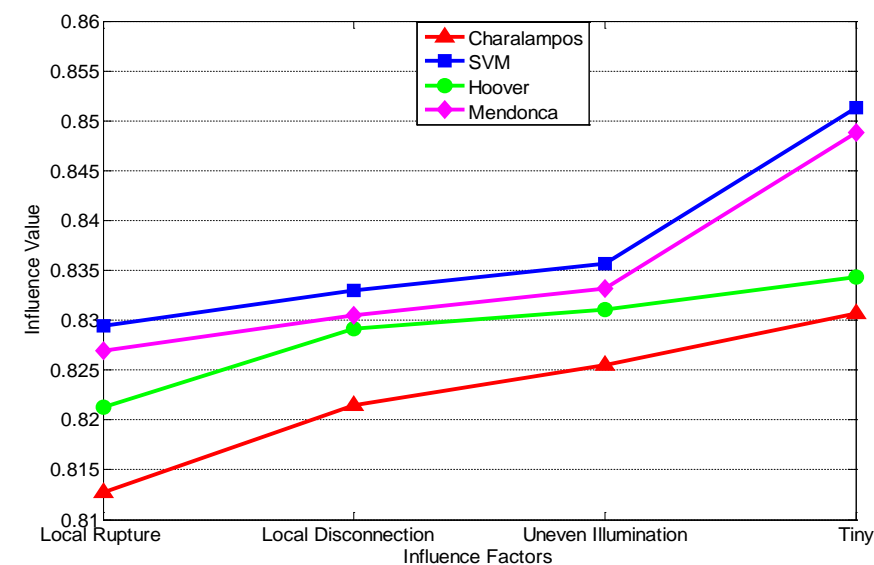

Fig.5 Comparison of the sensitivity of four algorithms on four factors

From the Fig.5, the sensitivity of different algorithms to different factors is clear. Table 2 shows the final recognition superiority. Considering the analysis of all kinds of factors, the superiority of the algorithm can be concluded as the following: Charalampos $<$ Hoover $<$ Mendonca $<$ SVM.

Table 2 the sensitivity of different algorithms to different factors

\begin{tabular}{lllll}
\hline & Rupture & Disconnection & Illumination & Tiny \\
\hline Charalampos & $\times$ & $\times$ & $\times$ & $\sqrt{-}$ \\
Hoover & $\times$ & $\sqrt{ }-$ & $\sqrt{ }-$ & $\times$ \\
Mendonca & $\sqrt{ }+$ & $\sqrt{ }-$ & $\sqrt{ }-$ & $\sqrt{ }-$ \\
SVM & $\sqrt{ }-$ & $\sqrt{ }-$ & $\sqrt{ }+$ & $\sqrt{ }+$ \\
\hline
\end{tabular}

$\times$ : fail, $\sqrt{ }-:$ medium, $\sqrt{ }+$ : good

\section{Conclusions}

A large amount of algorithms for CAM vessel segmentation have been published, yet an evaluation of these algorithms on a common database of CAM images has not been performed. As to common evaluation methods just take recognition quality into account that ignored the influence of different factors on the CAM images, so the evaluation result is poorly rough.

With the CAM image database consisting of four image sets, this new evaluation method can detect the sensitivity and accuracy of different algorithms to different factors that is local disconnection, local rupture, uneven illumination and tiny. So, the proposed evaluation method can accurately and sensitively 
determine the performance of different algorithms for CAM blood vessels under various conditions.

\section{Acknowledgements}

This study is supported by the National Natural Science Foundation of China (No. 61402100) and the Fundamental Research Funds for the Central Universities (14D111210).

\section{References}

[1] R. Jain. Molecular regulation of vessel maturation [J], Nat Med , 2003,9(1):685-693.

[2] Leng T, Miller JM, Bilbao KV. The chick chorioallantoic membrane as a model tissue for surgical retinal research and simulation [J]. Retina-the journal of retinal and vitreous diseases. 2004,3(24): 427-434 .

[3] Reed J, Correa R, Ramos-Valdes Y, et al. Establishment of the chick chorioallantoic membrane (CAM) as an ex vivo model system to study mechanisms underlying epithelial ovarian tumour growth and metastasis[J]. Cancer Research, 2011, 71(8 Supplement): 4309-4309.

[4] Nowak-Sliwinska P, Segura T, Iruela-Arispe M L. The chicken chorioallantoic membrane model in biology, medicine and bioengineering[J]. Angiogenesis, 2014, 17(4): 779-804.

[5] Hoover A, Kouznetsova V, Goldbaum M. Locating blood vessels in retinal images by piecewise threshold probing of a matched filter response [J]. IEEE Transactions on Medical Imaging, 2000,19(3):203-210.

[6] Hoover A, M. Goldbaum. Locating the optic nerve in a retinal image using the fuzzy convergence of the blood vessels [J]. IEEE Transactions on Medical Imaging, 2003,8(22): 951-958.

[7] Staal J, Abramoff M D, Niemeijer M. Ridge-based vessel segmentation in color images of the retina [J]. IEEE Transactions on Medical Imaging , 2004, 4(23):501-509.

[8] Biometric Systems Lab, Pattern Recognition and Image Processing Laboratory, biometric Test Center.

[9] Sim T, Baker S, Bsat M. The CMU pose, illumination, and expression database [J]. IEEE Transactions on Pattern Analysis and Machine Intelligence, 2003,12(25):1615-1618

[10] Phillips PJ, Moon H, Rizvi SA. The FERET evaluation methodology for face recognition algorithms[J]. IEEE Transactions on Pattern Analysis and Machine Intelligence , 2000, 10(22):1090-1104.

[11] Zhang YJ. A survey on evaluation methods for image segmentation [J]. Pattern Recognition, 1996, 8(29):1335-1346.

[12]ZhongyuXu, FenHu, HongchengGuo, QuanshengDou.Support vector machi ne image segmentation algorithm applied to angiogenesis quantification[J]. Proceedings 2010 Sixth International Conference on Natural Computation (ICNC 2010), 2010, 10(2):928-931. 
[13] Doukas, Charlampos N, Maglogiannis Ilias, Chatziioannou Aristotle. Automated angiogenesis quantification through advanced image processing techniques [J]. IEEE Engineering in Medicine and Biology Society, 2006,6(1):2345-2348.

[14] Mendonca A, Campilho A. Segmentation of retinal blood vessels by combining the detection of centerlines and morphological reconstruction. IEEE Transactions on Medical Imaging, 2006, 25(9):1200-1213. 